\title{
Structure Determination of Biomolecules by 3D Electron Diffraction
}

\author{
H. Xu and X. Zou \\ Department of Materials and Environmental Chemistry, Stockholm University, Sweden \\ Hongyi.xu@mmk.su.se
}

Knowing the 3D atomic structures of materials or biomolecules is crucial for understanding their functions. X-ray diffraction is currently the most important technique for determination of 3D atomic structures but requires large crystals which are often difficult to obtain. Electrons, similar to X-rays and neutrons, are powerful source for diffraction experiments. Due to the strong interactions between electrons and matter, crystals that are considered as powder in X-ray crystallography can be treated as single crystals by $3 \mathrm{D}$ electron diffraction methods [1]. This enables structure determination of materials and organic molecules from micron- to nanometersized 3D crystals that are too small for conventional X-ray diffraction. Furthermore, by taking the advantages of the unique properties of electron scattering, it is possible to determine the charge states of atoms/ions [2] and the absolute structure of chiral crystals [3].

Over the past decades, a number of 3D ED methods have been developed for structure determination. At the early stages of 3D ED method development, tilting of the crystal was done manually, while diffraction patterns were collected on negative film. It could take years before sufficient data were obtained and processed in order to determine the crystal structure. The computerization of TEMs and the development of CCD detectors allowed software to be developed that can semi-automatically collect 3D ED data in less than an hour [1]. Thanks to the recent advancement in CMOS and hybrid detector technology, it is now feasible to collect diffraction data in movie mode while continuously rotating the crystal (continuous rotation election diffraction, cRED, also known as MicroED [4] in structural biology). Benefiting from these technological advances, structure determination can now be accomplished within a few hours. Recently, fully automated serial rotation electron diffraction data collection and processing has been realized by our group [5].

By using 3D ED / MicroED methods, we have solved more than 200 novel crystal structures of small inorganic compounds [6] (including zeolite, MOF, COF and minerals) and biomolecules [7,8] (pharmaceuticals, small organic molecules, peptides and proteins) in the past 7 years. Recently, we have solved two novel protein $[9,10]$ structures with $3 \mathrm{D}$ ED/MicroED and shown that it is feasible to use MicroED for structure-based drug discovery [11]. We aim to further improve these methods, develop new methods and more importantly spread them to labs around the world.

[1] Gemmi M., Mugnaioli E., Gorelik T. E., Kolb U., Palatinus L., Boullay P., Hovmöller S. \& Abrahams J. P. (2019). ACS Cent. Sci. 5, 13151329.

[2] Yonekura K., Kato K., Ogasawara M., Tomita M. \& Toyoshima C. (2015). Proc. Natl. Acad. Sci. 112, 3368-3373.

[3] Brázda P., Palatinus L. \& Babor M. (2019). Science. 364, 667-669.

[4] Shi D., Nannenga B. L., Iadanza M. G. \& Gonen T. (2013). eLife. 2, e01345.

[5] Wang B., Zou X. \& Smeets S. (2019). IUCrJ. 6, 854-867.

[6] Huang Z., Willhammar T. \& Zou X. (2021). Chem. Sci. 12, 1206-1219.

[7] Clabbers M. T. B. \& Xu H. (2020). Drug Discov. Today Technol., S1740674920300354.

[8] Clabbers M. T. B. \& Xu H. (2021). Acta Crystallogr. Sect. Struct. Biol. 77, 313-324.

[9] Xu H., Lebrette H., Clabbers M. T. B., Zhao J., Griese J. J., Zou X. \& Högbom M. (2019). Sci. Adv. 5, eaax4621.

[10] Clabbers M. T. B., Holmes S., Muusse T. W., Vajjhala P., Thygesen S. J., Malde A. K., Hunter D. J. B., Croll T. I., Flueckiger L., Nanson J. D., Rahaman H., Aquila A., Hunter M. S., Liang M., Yoon C. H., Zhao J., Zatsepin N. A., Abbey B., Sierecki E., Gambin Y., Stacey K. J., Darmanin C., Kobe B., Xu H. \& Ve T. Nat. Commun. (In Press)

[11] Clabbers M. T. B., Fisher S. Z., Coinçon M., Zou X. \& Xu H. (2020). Commun. Biol. 3, 417.

\section{Keywords: 3D Electron Diffraction; MicroED; Structure Determination; Protein; Pharmaceutics}

The authors would like to acknowledge the contribution made by the PhD students and postdocs in the group. The project is supported by the Knut and Alice Wallenberg Foundation (2018.0237, X.Z.), the Swedish Research Council (2017-05333, H.X.; 2019-00815, X.Z.) and the Science for Life Laboratory through the pilot project grant Electron Nanocrystallography, and MicroED@SciLifeLab. 